
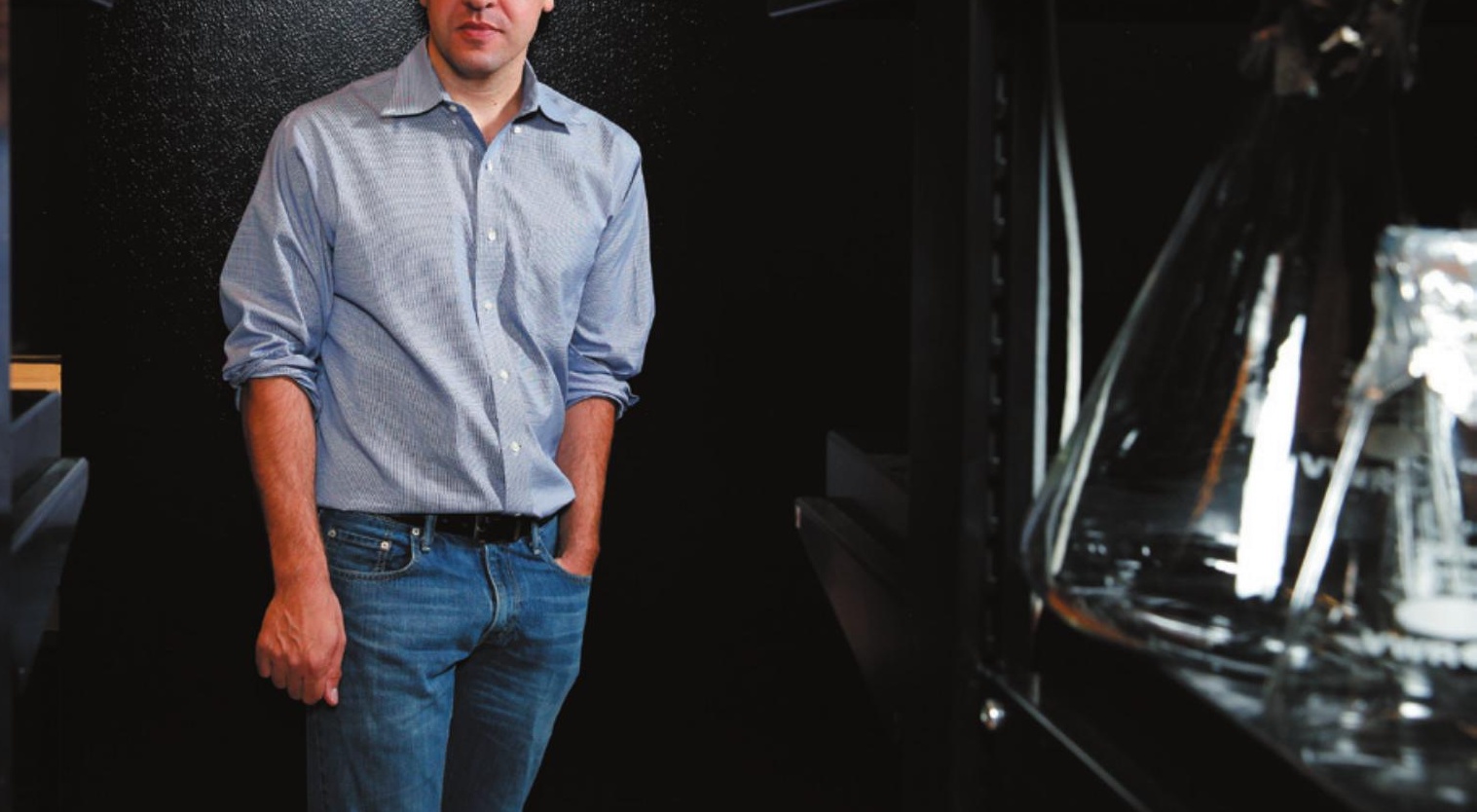

\title{
Method math
}

BY KERRI SMITH

$\mathrm{W}$ hen Karl Deisseroth moved into his first lab in 2004, he found himself replacing a high-profile tenant: Nobel-prizewinning physicist Steven Chu. "His name was still on the door when I moved in," says Deisseroth, a neuroscientist, of the basement space at Stanford University in California. The legacy has had its benefits. When chemistry student Feng Zhang dropped by looking for Chu, Deisseroth convinced him to stick around. "I don't think he knew who I was. But he got interested enough."

Deisseroth is now a major name in science himself. He is associated with two blockbuster techniques that allow researchers to show how intricate circuits in the brain create patterns of behaviour. The development of the methods, he says, came from a desire to understand mechanisms that give rise to psychiatric disease - and from the paucity of techniques to do so. "It was extremely clear that for fundamental advances in these domains I would have to spend time developing new tools," says Deisseroth.

His measured tone and laid-back demeanour belie the frenzy that his lab's techniques are generating in neuroscience. First came optogenetics ${ }^{1}$, which involves inserting lightsensitive proteins from algae into neurons, allowing researchers to switch the cells on and off with light. Deisseroth developed the method shortly after starting his lab, working with Zhang and Edward Boyden, a close collaborator at the time. Optogenetics has since been adopted by scientists around the world to explore everything from the functions of neuron subtypes to the circuits altered in depression or autism. Deisseroth has lost count of how many groups are using it. "We sent clones 
to thousands of laboratories," he says.

Now his lab is gearing up for another rush, after publishing a method called CLARITY ${ }^{2}$. The technique uses a chemical treatment to turn whole brains transparent, allowing researchers to examine the brain's structure in detail in three dimensions. It could help researchers to connect the brain's function with its structure. "I think it'll be de rigueur for people to have a picture showing the pathways and how they sit in the brain," says Richard Tsien, the neuroanatomist under whom Deisseroth earned his $\mathrm{PhD}$ at Stanford.

Deisseroth takes risks and encourages his team to do the same, often pulling together people with varying expertise and keeping abreast of many different fields. Christof Koch, chief scientific officer at the Allen Institute for Brain Science in Seattle, Washington, puts Deisseroth's success down to these "catholic interests". But Deisseroth also has a laser-like focus on tool-building, fuelled, he says, by his work as a practising psychiatrist. And although he is excited about his lab's newest technique, Deisseroth plays it down, shrugging a broad shoulder: "I'm not very demonstrative," he says.

\section{TAKING CONTROL}

When Tsien, who now works at New York University's Langone Medical Center, first met Deisseroth, he was struck by his determination. "It was an unusual encounter - he came for an interview and told me rather shyly that the reason he came to Stanford was because he wanted to work with me," Tsien remembers. It was 1993 and Tsien's lab was "crammed to the gills", he says, but Deisseroth was persistent and persuasive. As part of a joint programme to earn a $\mathrm{PhD}$ and a medical doctorate, he began a project looking at calcium channels in neurons. In 2000, he followed that up with a multi-pronged appointment: a postdoctoral fellowship in the lab of Stanford neuroscientist Robert Malenka and - after toying with the idea of neurosurgery - a residency in psychiatry at Stanford Medical School.

"Everything changed when I did my psychiatry rotation," says Deisseroth. "A person can be right in front of you who looks intact, not obviously injured, and yet their brain is constructing for them a completely different reality. At the same time I saw how deep the suffering was."

Studying depression or anxiety in a dish of cells was inadequate, he reasoned, because only whole brains can give rise to the sophisticated functions - and disorders - that characterize human behaviour. And techniques for studying whole brains in humans and model organisms were often limited to simply watching them at work.

So Deisseroth began thinking about ways to examine and control intact systems. "I was having a lot of discussions with a lot of people," he says. During his residency, Deisseroth met

Boyden, a PhD student with similarly ambitious aims. The two began talking about ways to manipulate individual neurons as a side project. "It was a very adventurous collaboration, full of exploration," says Boyden.

One idea involved using light to control neuronal firing. Boyden and Deisseroth knew about light-sensitive channel proteins called opsins, which algae use for generating energy,

\section{"It was a little hard} for people in the field to understand what he was trying to do."

among other functions. Several groups including that of Tsien's brother Roger at the University of California, San Diego - were trying to insert these proteins into neuron cell membranes. The project needed "the wherewithal to spend the money and find the graduate students", says Deisseroth. In 2004, having secured his own lab, he could do just that.

By July of that year, Deisseroth had managed to coax neurons into expressing opsins in their cellular membranes. Using cells borrowed from Malenka and recording equipment from Richard Tsien, he, Zhang and Boyden hunkered down to see if the technique would fly. "It worked on pretty much the first try," Boyden recalls. "It was like riding a wave of serendipity."

In the following year, Deisseroth finally secured funding from the US National Institutes of Health (NIH) to further the work. He had faced multiple rejections. Projects based on technologies have a harder time attracting support than hypothesis-driven projects, says Thomas Insel, director of the US National Institute for Mental Health in Bethesda, Maryland, the source of the initial federal funding for Deisseroth's optogenetics research. "It was a little hard for people in the field to understand what he was trying to do," says Insel.

But neuroscientists saw the potential in 2005, when Deisseroth's group published its first big paper showing that the technique worked in a dish ${ }^{1}$. Researchers could now stimulate a specific type of neuron and see how that affected the cell's - or even an entire animal's - behaviour. Requests for the technology were pouring in.

The technique has since been used for everything from studying the development of neural stem cells ${ }^{3}$ to prompting mice to recall fearful memories $^{4}$ (see Nature 465, 26-28; 2010). Deisseroth and his team, with their focus on psychiatric conditions, have used rodent models

$\rightarrow$ NATURE.COM For interviews with Deisseroth anda video of CLARITY, see: go.nature.com/zsxdld to explore the network of brain areas that gives rise to anxiety and have shown that one 'hub' controls diverse symptoms such as an elevated breathing rate and feelings of panic and discomfort ${ }^{5}$. They have switched on and off the activity of mouse neurons that use the neurotransmitter dopamine, to show how they contribute to the symptoms of depression ${ }^{6}$. The team has even used optogenetics to prevent cocaine-addicted rats from seeking out the $\mathrm{drug}^{7}$. The work, says Deisseroth, could help scientists to design antianxiety drugs that are less addictive than current treatments - affecting anxiety pathways but leaving reward circuitry untouched.

In 2006, Boyden started his own lab at the Massachusetts Institute of Technology in Cambridge. Rumours emerged that his relationship with Deisseroth was souring. Neither is keen to discuss the details, but in 2007, the two groups published separate papers on halorhodopsin, an 'off-switch' opsin that they had previously studied together ${ }^{8,9}$. "We didn't know Ed was working on that until we sent our paper to Nature," says Zhang. "Well you know, in any competitive scenario people are interested in being first," says Boyden.

The experience might have left its mark on Deisseroth: he is careful to attribute credit precisely to his team members and trainees, particularly with respect to CLARITY. "Karl is quite sensitive about who came up with the idea," says Kwanghun Chung, first author of the CLARITY paper ${ }^{2}$. Creating see-through brains was Deisseroth's dream, but the chemical know-how came from Chung. They describe the finding as a co-discovery.

\section{SEEING CLEARLY}

Standard techniques for examining fine-scale brain structure involve slicing the brain into tissue-thin segments, analysing them under a microscope, and then - laboriously and often imprecisely — stacking the images back together. To bypass that process, Deisseroth had been looking for a means of chemically treating the brain to make it transparent. Some components of brain cells - especially lipids - are notoriously opaque to microscopes, so the idea was to get rid of them, while preserving the neuronal structure. It was a very different approach from optogenetics, and recruiting Chung - a chemical engineer by background - displayed Deisseroth's willingness to take risks. "I remember people wondering what I was doing, what sort of plan there was in hiring this person and the direction the lab was going, because it was such a marked shift," he says.

At first, Deisseroth wanted to immobilize neurons by filling them with a suitable material and removing the surrounding tissue. It turned out to be very difficult to build a stable structure from inside the neurons, but it was easier to build one around them.

The team tried a few scaffolding materials, including keratin and a cellulose-like structure, before settling on a hydrogel - a gel made mainly of water and already widely used in biological studies. The researchers found that a 

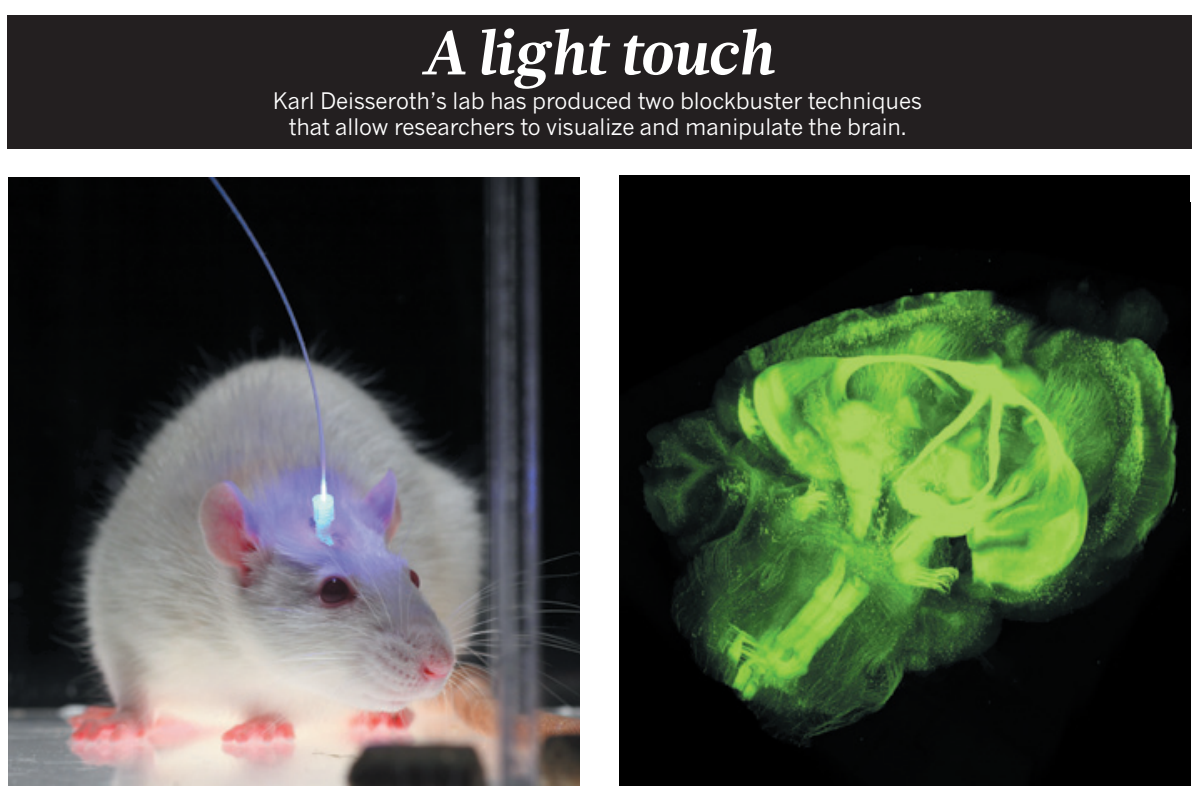

\section{Optogenetics}

1

are used to ferry genes encoding ght-sensitive receptors (opsins) into specific neurons

Animals are fitted with an 'optrode', a fibre-optic cable with an electrode.

Light beamed down the optrode will open or close ion channels while the electrode records neuronal firing and researchers record behaviours.

hydrogel scaffold could bind components of neurons in place, including proteins, neurochemicals and even DNA and RNA (see 'A light touch'). With the structure locked down, a detergent called SDS could wash away lipid membranes, leaving the tissue transparent (see Nature 496, 151; 2013).

Within a week of publication, Deisseroth had already received several dozen requests for information. "We've been very much... I'm trying to find a verb that doesn't make it seem like a problem," he says. He settles on describing the e-mails that the lab received as an "outpouring".

CLARITY stunned people in the same way that optogenetics did, says Insel. "Usually when someone has at an early stage of their career made an important advance, they tend to rest on those laurels. Karl quite quickly decided to do the next big thing."

Other researchers will tailor CLARITY to their own projects. David Van Essen, a neuroscientist at Washington University in St. Louis, Missouri, is interested in testing CLARITY in the brain's white matter, the bundles of neuronal projections called axons that carry nerve impulses. This will help his group study patterns of connectivity that link brain regions.

Using CLARITY with techniques such as optogenetics, or using it to analyse brains after behavioural studies, will be a powerful way to extract information about how brain networks
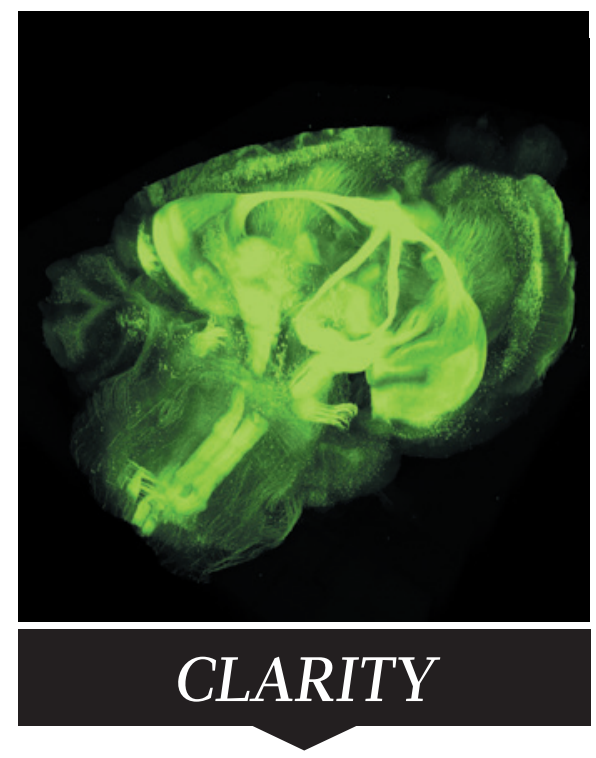
are hybridized to a hydrogel matrix, fixing them in place.

2 Densely packed, light-blocking lipids are washed away using detergents.

The clarified brain can be stained and washed multiple times, allowing scientists to observe different molecules and cell types.

function, says Eve Marder, a neuroscientist at Brandeis University in Waltham, Massachusetts. But analysing large networks in detail is challenging. Marder usually works with simple circuits of 30 or so neurons, and says that even those have a scarily exponential number of permutations. "My only personal hope is that peobrains pay attention to the lessons learned and mistakes that we made along the way," she says.

CLARITY is already shedding light on clinical disorders. In the paper describing the technique $^{2}$, the researchers analysed brain tissue from a seven-year-old boy who had autism spectrum disorder. They found that neurons in his cortex had joined together in ladderlike patterns, rather than the branches seen in typical brains. Animal models of autism-like conditions had hinted at this difference, but CLARITY made it possible to look for the irregularity in human brain samples.

\section{RISK AND REWARD}

Deisseroth's office and much of his lab are still in the same basement that he moved into in 2004, although the team - now including 35 people - has branched out into lab space elsewhere. The labs are strewn with gadgetry, like the bedroom of a technology-obsessed teenager. There is no natural light in the basement, but Deisseroth likes it; being at the bottom of
1 Proteins and other molecules in the brain ple who venture into doing circuit work in larger the building keeps vibrations to a minimum, which is necessary when using the sophisticated microscopes essential for his work.

The current team includes computationa neuroscientists, medics, chemists and engineers. And a US\$22.5-million Transformative Research Award from the NIH, granted last year, allows Deisseroth to keep encouraging them to take chances. "I have everyone have a hand in a high-risk and a low-risk project," he says, "like a hedged portfolio."

One of the latest high-risk projects uses light-field microscopy, which records images from a variety of perspectives simultaneously, to image the brain. Elsewhere in the lab Chung and collaborator Viviana Gradinaru are still trying to build hydrogel structures inside neurons in the hope of preserving networks of specific cell types - freezing in place all the neurons of a certain type, for example, or all the cells that express a particular gene. The team continues to improve optogenetics, too, developing new opsins and perfecting methods for delivering light to the brain.

Deisseroth has already added space to the laboratory to cater for all the visiting scientists coming to learn how to use optogenetics and CLARITY. There were more than 200 in 2010-12. The group has “done yeoman's work in making this relatively straightforward to set up", says Van Essen. "They're not going to hold hostage a methodology that people really can set up in their own lab." The tools are freely available; Stanford has filed patents on some aspects, says Deisseroth, but that it is to ensure unfettered access to the technology. He does not make money from the patents.

Deisseroth struggles with his decision to spend more time on research than with the patients who inspire it. He still runs a weekly psychiatry clinic, but has to balance this with a packed travel schedule and mentoring duties. He carries on, however, hopeful that his studies will ultimately benefit those that medicine cannot. "We can help patients right now with many psychiatric symptoms," he says. "But for others we can't. So that really helps me in mentally freeing up the time needed for research."

That desire to help is buoyed by simple, boundless curiosity. "I think all scientists have a little bit in them," he says. "That wonder and curiosity about the natural world - not only how it is, but how it could be."

Kerri Smith is senior audio editor for Nature in London.

1. Boyden, E. S., Zhang, F., Bamberg, E., Nagel, G. \& Deisseroth, K. Nature Neurosci. 8, 1263-1268 (2005).

2. Chung, K. et al. Nature 497, 332-337 (2013)

3. Stroh, A. et al. Stem Cells 29, 78-88 (2011).

4. Liu, X. et al. Nature 484, 381-385 (2012)

5. Kim, S.-Y. et al. Nature 496, 219-223 (2013)

6. Chaudhury, D. et al. Nature 493, 532-536 (2013).

7. Stefanik, M. T. et al. Addiction Biology 18, 50-53 (2013).

8. Han, X. \& Boyden, E. S. PLoS ONE 2, e299 (2007)

9. Zhang, F. et al. Nature 446, 633-639 (2007). 